\title{
NOUVELLE
}

\section{Une nouvelle génération d'immunothérapies ciblant la voie CD39/CD73/adénosine pour favoriser la réponse immunitaire anti-tumorale}

Laurent Gros ${ }^{1}$, Carine Paturel $^{2}$, Ivan Perrot ${ }^{2}$, Armand Bensussan ${ }^{3,4}$, Jean-François Eliaou ${ }^{1,5}$, Jérémy Bastid ${ }^{3}$, Nathalie Bonnefoy ${ }^{1,3}$

\footnotetext{
${ }^{1}$ Institut de recherche en cancérologie de Montpellier, Inserm U1194, université de Montpellier, institut régional du cancer de Montpellier, 34298 Montpellier, France.

${ }^{2}$ Innate Pharma, 117 avenue de Luminy, 13009 Marseille, France.

${ }^{3}$ OREGA Biotech, 69130 Ecully, France.

${ }^{4}$ Inserm UMRS 976 , université Paris Diderot, Sorbonne Paris Cité, laboratoire immunologie, dermatologie et oncologie, 75475 Paris, France.

${ }^{5}$ Département d'immunologie, centre hospitalier régional universitaire de Montpellier et faculté de médecine, université de Montpellier, 34295 Montpellier, France. nathalie.bonnefoy@inserm.fr
}

$>$ Le concept d'immunosurveillance et la compréhension des mécanismes par lesquels le système immunitaire peut éradiquer des tumeurs ont conduit au développement de nouvelles formes d'immunothérapie du cancer visant à promouvoir ou rétablir une réponse immunitaire anti-tumorale efficace et durable. Cette idée révolutionnaire de cibler, non plus les cellules tumorales, mais les cellules immunitaires, est à l'origine d'avancées médicales majeures. Des résultats sans précédent ont en effet été obtenus en utilisant des inhibiteurs des points de contrôle du système immunitaire (immune checkpoint inhibitors, $\mathrm{ICl}$ ) tels que les anticorps anti-PD-1, anti-PD-L1, et anti-CTLA-4, pour traiter des cancers réfractaires aux traitements conventionnels par chimiothérapie, radiothérapie, ou thérapie ciblée, dont le pronostic était jusqu'alors très défavorable [1-3]. Ces nouvelles immunothérapies, utilisées seules ou en combinaison, font désormais partie de l'arsenal thérapeutique contre un grand nombre de cancers, et ont permis d'obtenir des rémissions complètes. Toutefois, les effets bénéfiques durables ne concernent qu'une minorité des patients traités ( $20 \%$ à $30 \%$ tous cancers confondus), tandis que les autres patients présentent une résistance innée ou acquise aux $\mathrm{ICI}$ [4]. La mise au point de traitements combinant immunothérapie et radiothérapie ou chimiothérapie, mais aussi l'identification de nouvelles cibles afin d'améliorer l'efficacité des $\mathrm{ICI}$ sont au cœur des nouvelles stratégies 


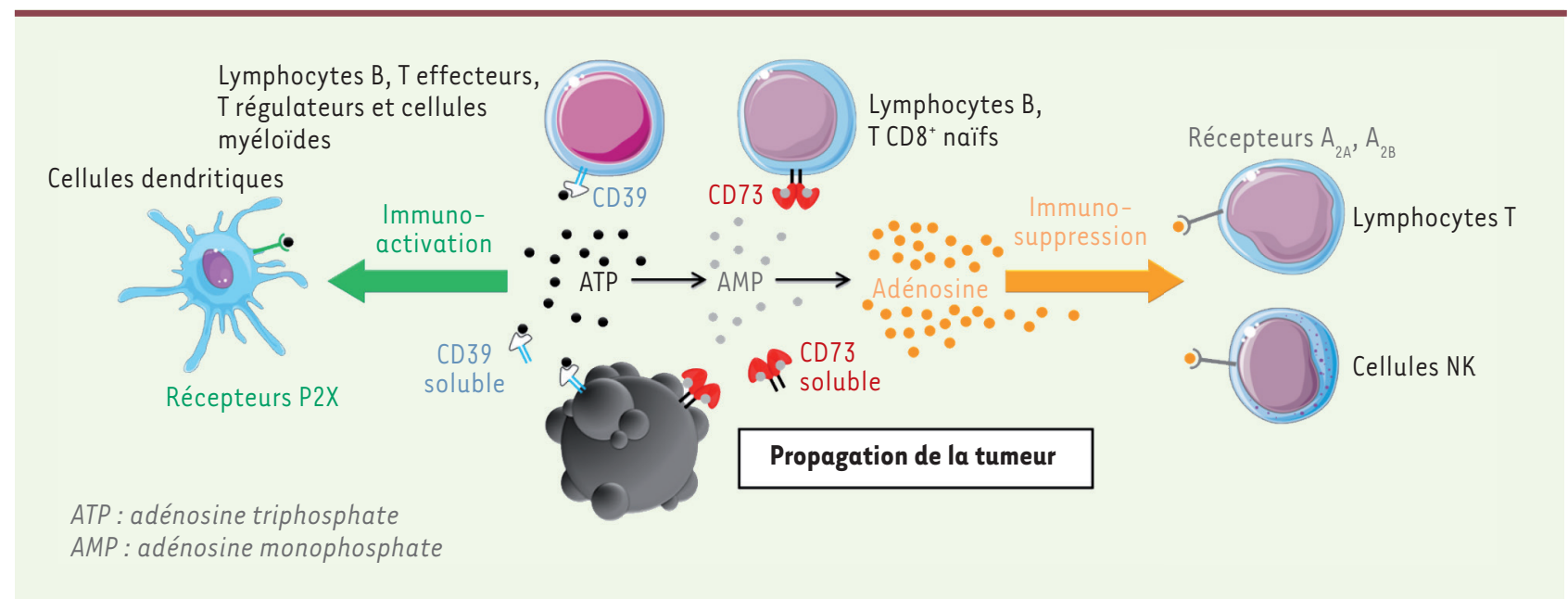

Figure 1. La voie ATP/AMP/adénosine. Les exoenzymes CD39 et CD73 favorisent l'accumulation d'adénosine dans le microenvironnement tumoral et inhibent les réponses anti-tumorales. CD39 et CD73 sont exprimées par certaines cellules immunes infiltrant la tumeur et par les cellules tumorales elles-mêmes. Elles transforment l'ATP extracellulaire, relargué par les cellules tumorales mourantes, en AMP, puis en adénosine. L'adénosine, en se fixant sur les récepteurs $A_{2 A}$ et $A_{2 B}$, inhibe les fonctions effectrices des lymphocytes T CD8 ${ }^{+}$et des cellules NK. La diminution de la quantité d'ATP, quant à elle, diminue l'activation des cellules dendritiques via les récepteurs P2X. Ces deux actions concomitantes favorisent la prolifération et la dissémination des cellules tumorales.

thérapeutiques contre le cancer. Dans ce contexte, le ciblage des voies d'immunosuppression du microenvironnement de la tumeur constitue l'un des enjeux. Les résultats de nos récents travaux de recherche, publiés dans Cell Reports [5], fournissent le rationnel scientifique pour le développement clinique de deux anticorps ciblant les protéines CD39 et CD73, et sont en faveur de leur utilisation dans l'immunothérapie des cancers.

\section{CD39, CD73, et adénosine : de} nouvelles cibles thérapeutiques contrôlant la réponse immunitaire anti-tumorale

L'adénosine, par ses propriétés immunosuppressives, est un acteur majeur de l'échappement des tumeurs au contrôle par le système immunitaire. Ce nucléoside joue un rôle essentiel dans la migration, la prolifération, et la mort cellulaire [6]. C'est un frein puissant à la réponse immunitaire par sa capacité à inhiber l'activation des cellules dendritiques, la prolifération et l'activation des lymphocytes T effecteurs, l'activation des cellules natural killer (NK), ou au contraire en activant les fonctions suppressives des lymphocytes T régulateurs et des macrophages pro-tumoraux de type M2 [6]. La production d'adénosine dans le microenvironnement tumoral est liée à l'activité de deux ectonucléotidases, CD39 (ectonucléoside triphosphate diphosphohydrolase 1, ENTPD1) et CD73 (ecto-5'-nucléotidase, NT\&5). Une augmentation de l'expression de CD39 et CD73 dans le lit tumoral témoigne d'un environnement immunosuppresseur.

CD39, sous forme membranaire ou soluble, hydrolyse l'adénosine triphosphate (ATP) et l'adénosine diphosphate $(A D P)$ en adénosine monophosphate (AMP). L'AMP est converti à son tour en adénosine par CD73. À l'inverse de l'adénosine, I'ATP extracellulaire libéré lors de la mort des cellules tumorales agit comme un signal immunogénique puissant [7]. L'ATP, en se liant aux récepteurs membranaires purinergiques $P 2 X$ et $P 2 Y$, favorise la maturation des cellules dendritiques et l'amorçage (priming) de la réponse anti-tumorale. L'ATP inhibe la prolifération des cellules tumorales et favorise leur mort. Le rapport ATP/adénosine est donc un élément important pour la fonction anti- tumorale du système immunitaire, et les enzymes CD39 et CD73 apparaissent comme des enzymes clefs, dont l'activité séquentielle contrôle la concentration d'ATP (immunostimulatrice) et d'adénosine (immunosuppressive) [8] (Figure 1). Confortant l'hypothèse du rôle de CD39 et CD73 dans le contrôle de la réponse immunitaire anti-tumorale, plusieurs études montrent que les souris déficientes en CD39 ou CD73 ont moins de métastases et une croissance de tumeurs syngéniques transplantées diminuée [9-11]. Par ailleurs, le blocage de l'activité de l'une ou l'autre de ces ectonucléotidases par des inhibiteurs chimiques ou des anticorps monoclonaux a produit des effets encourageants dans des études pré-cliniques [12-14].

\section{Neutralisation de l'activité ATPasique} de CD39 : impact sur l'efficacité des thérapies anti-tumorales

$\varepsilon$ n utilisant trois modèles murins (mélanome Bl6Flo, fibrosarcome MCA205, et adénocarcinome colorectal MC38), nous avons montré que l'invalidation du gène codant CD39 chez la souris est associée à une augmentation 


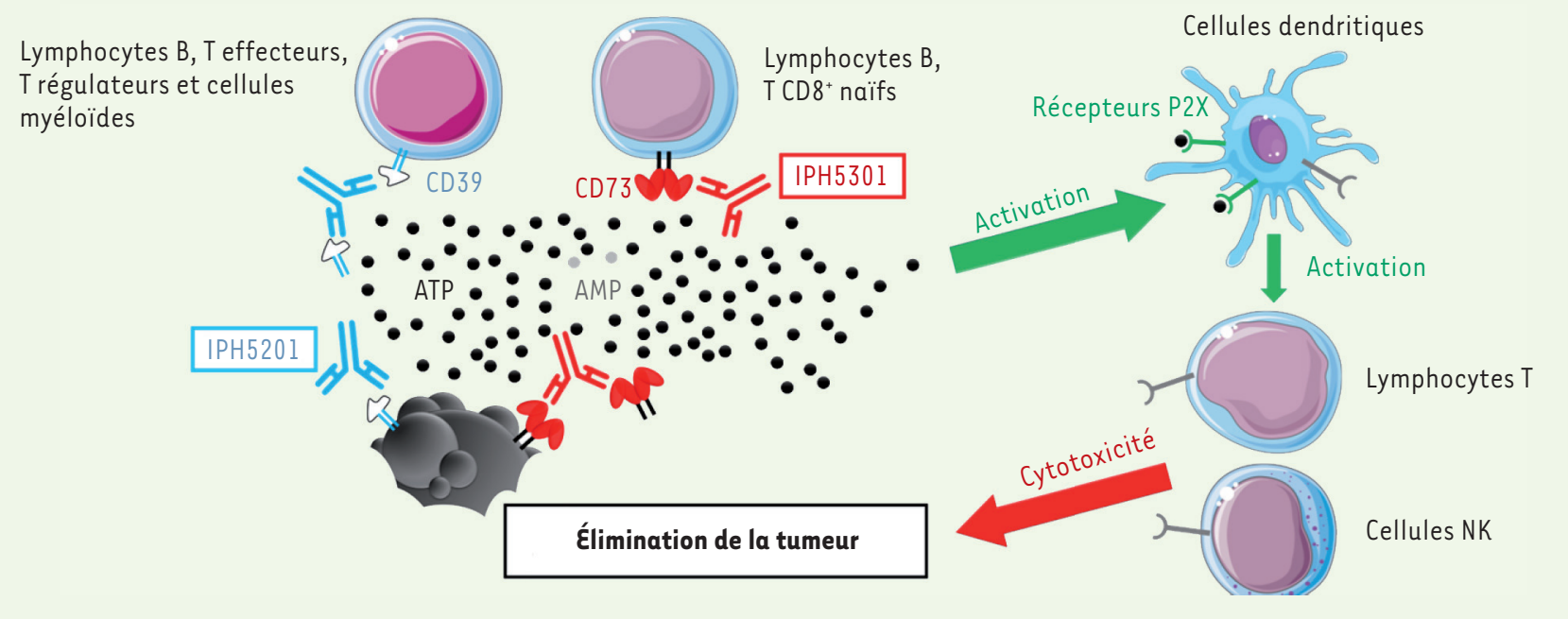

Figure 2. Mécanisme d'action des anticorps IPH5201 et IPH5301. Les anticorps IPH5201 et IPH5301 bloquent respectivement l'activité enzymatique de CD39 et celle de CD73, donc l'hydrolyse de l'ATP et de l'AMP extracellulaire. Ces deux actions conjointes permettent, d'une part de limiter la production d'adénosine et ainsi l'inhibition des cellules immunes exprimant les récepteurs de l'adénosine (récepteurs $A_{2 A}, A_{2 B}$ ), et, d'autre part, de potentialiser l'activation des cellules dendritiques via les récepteurs P2X. L'activation optimale des cellules immunes infiltrant la tumeur permet la mise en place de réponses anti-tumorales efficaces et l'élimination de la tumeur.

des fonctions effectrices des lymphocytes $\mathrm{TCD}^{+}{ }^{+}$et $\mathrm{CD} 4^{+}$infiltrant la tumeur et à un retard de la progression tumorale. Cependant, l'invalidation du gène codant CD39 ne permet à elle seule la régression complète des tumeurs dans aucun de ces trois modèles. En revanche, elle potentialise le taux de réponse favorable aux $\mathrm{ICl}$ anti-PD-1 et anti-CTLA-4, et permet une rémission complète (élimination des tumeurs) et durable chez $20 \%$ des animaux traités par un anticorps anti-PD-1 dans le modèle de mélanome $\mathrm{B} 16 \mathrm{Fl}$, particulièrement résistant à ces immunothérapies. Dans le modèle de fibrosarcome MCA205, le traitement de souris déficientes en CD39 par l'anticorps antiPD-1 seul retarde légèrement la croissance tumorale, mais n'induit pas de rémission complète. Cependant, dans ce modèle, le taux de rémission complète obtenu avec la combinaison de I'oxaliplatine et de l'anticorps antiPD-1 passe de $65 \%$ chez les souris témoins à plus de $90 \%$ chez les souris déficientes en CD39. Cet effet est strictement dépendant des lymphocytes $T$ $\mathrm{CD} 8^{+}$et $\mathrm{CD}^{+}$, et est associé au déve- loppement d'une mémoire immunitaire protectrice. Ces résultats montrent que la neutralisation de l'activité ATPasique de CD39 chez la souris potentialise l'efficacité des traitements anticancéreux utilisant des $\mathrm{ICI}$ combinés ou non à une chimiothérapie, et augmente significativement les taux de réponse complète et de survie. Ils ouvrent ainsi des perspectives pour de nouvelles approches de thérapies combinées.

\section{Inhibition de CD39 et de CD73 :} redondance ou synergie ?

Le développement concomitant d'anticorps bloquant CD39 (IPH5201) ou CD73 (IPH5301) nous a permis d'aborder cette question pour la première fois. L'anticorps IPH5201 inhibe l'activité enzymatique de CD39 membranaire et soluble, et bloque efficacement l'hydrolyse de I'ATP extracellulaire. Son utilisation augmente le taux de réponse à l'oxaliplatine des souris porteuses du fibrosarcome MCA205 et invalidées pour le gène codant CD39 mais exprimant la protéine CD39 humaine, alors que son utilisation en monothérapie n'affecte pas la croissance tumorale chez ces souris.
L'anticorps IPH5301 bloque la dégradation de l'AMP en adénosine par CD73, et son affinité pour cette enzyme est similaire à celle de deux anticorps de référence, MEDI9447 et CD73.4, actuellement en cours d'essais cliniques de phase Ib/II et I/Ila. Cependant, IPH5301 est plus efficace pour bloquer l'activité enzymatique de CD73 sous forme membranaire ou soluble et pour restaurer la prolifération des lymphocytes $T$ in vitro.

Ces anticorps IPH520l et IPH5301, lorsqu'ils sont utilisés seuls, bloquent efficacement la production d'adénosine et restaurent in vitro la prolifération des lymphocytes du sang périphérique de sujets sains ou de patientes atteintes d'un cancer du sein, dans un milieu riche en ATP. Remarquablement, dans les mêmes expériences in vitro, lorsqu'ils sont utilisés ensemble à des doses qui étaient insuffisantes pour restaurer la prolifération des lymphocytes quand ils étaient utilisés seuls, IPH5201 et IPH5301 agissent en synergie pour supprimer l'effet suppressif lié à la dégradation de l'ATP en adénosine et pour favoriser la prolifération des 
lymphocytes T. Ainsi, les blocages de l'activité enzymatique de CD39 et CD73 n'apparaissent pas redondants, mais synergiques pour limiter l'inhibition des lymphocytes $T$ dépendante de l'adénosine et améliorer ainsi l'immunité antitumorale (Figure 2). $\diamond$

A new generation of immunotherapies targeting the CD39/CD73/adenosine pathway to promote the anti-tumor immune response

\section{LIENS D'INTÉRÊT}

$A B, J F E, J B$ et $N B$ sont actionnaires et/ou salariés d'Orega Biotech et inventeurs de brevets couvrant des anticorps anti-CD39. CP et IP sont salariés d'Innate Pharma et inventeurs de brevets couvrant des anticorps anti-CD39 ou anti-CD37. LG déclare n'avoir aucun lien d'intérêt concernant les données publiées dans cet article.

\section{RÉFÉRENCES}

1. Hodi FS, O'Day SJ, McDermott DF, et al. Improved survival with ipilimumab in patients with metastatic melanoma. N Engl J Med 2010 ; 363 : 711-23.

2. Topalian SL, Hodi FS, Brahmer JR, et al. Safety, activity, and immune correlates of anti-PD-1 antibody in cancer. $N$ Engl J Med 2012 ; $366: 2443-54$.

3. Brahmer JR, Tykodi SS, Chow LQ, et al. Safety and activity of anti-PD-Ll antibody in patients with advanced cancer. N Engl J Med 2012 ; 366 : 2455-65.

4. Jenkins RW, Barbie DA, Flaherty KT. Mechanisms of resistance to immune checkpoint inhibitors. $\mathrm{Br}$ J Cancer 2018; $118: 9-16$.

5. Perrot I, Michaud HA, Giraudon-Paoli M, et al. Blocking antibodies targeting the CD39/CD73 immunosuppressive pathway unleash immune responses in combination cancer therapies. Cell Rep 2019; 27 : 2411-25 e9.

6. de Andrade Mello P, Coutinho-Silva R, Savio LEB. Multifaceted effects of extracellular adenosine triphosphate and adenosine in the tumor-host interaction and therapeutic perspectives. Front Immunol $2017 ; 8$ : 1526.

7. Silva-Vilches C, Ring S, Mahnke K. ATP and its metabolite adenosine as regulators of dendritic cell activity. Front Immunol $2018 ; 9: 2581$.
8. Bastid J, Regairaz A, Bonnefoy N, et al. Inhibition of CD39 enzymatic function at the surface of tumor cells alleviates their immunosuppressive activity. Cancer Immunol Res $2015 ; 3: 254-65$.

9. Sun X, Wu Y, Gao W, et al. CD39/ENTPDl expression by $\mathrm{CD}^{+}$Foxp $3^{+}$regulatory $T$ cells promotes hepatic metastatic tumor growth in mice. Gastroenterol 2010 ; 139: 1030-40.

10. Stagg J, Divisekera U, Duret H, et al. CD73-deficient mice have increased antitumor immunity and are resistant to experimental metastasis. Cancer Res $2011 ; 71$ : 2892-900.

11. Stagg J, Beavis PA, Divisekera U, et al. CD73-deficient mice are resistant to carcinogenesis. Cancer Res $2012 ; 72$ : 2190-6.

12. Antonioli L, Yegutkin GG, Pacher P, et al. Anti-CD73 in cancer immunotherapy: awakening new opportunities. Trends Cancer 2016 ; 2 : 95-109.

13. Antonioli L, Novitskiy SV, Sachsenmeier KF, et al. Switching off CD73: a way to boost the activity of conventional and targeted antineoplastic therapies. Drug Discov Today 2017 ; 22 : 1686-96.

14. Nikolova M, Carriere M, Jenabian MA, et al. CD39/ adenosine pathway is involved in AIDS progression. PLoS Pathog 2011 ; 7 : el002110. 